\title{
EVOLUCIÓN DE LAS REPRESENTACIONES MENTALES SOBRE COLISIONES
}

\author{
VILLANI, A.' y ORQUIZA DE CARVALHO, L. ${ }^{2}$ \\ ${ }^{1}$ Instituto de Física. Universidade de São Paulo. Caixa Postal. São Paulo, SP, Brasil. \\ ${ }^{2}$ Faculdade de Engenharia. Universidade Estadual Paulista. Ilha Solteira, SP, Brasil.
}

\section{SUMMARY}

The article concerns the results of a qualitative research with secondary school students on the evolution of their ideas about collisions in mechanics. We focus our attention on the mechanism of partial attainment of new ideas and the relationship between the resistance to change and the experimental situations. Some implications for teaching are also discussed.

\section{INTRODUCCIÓN}

Las investigaciones sobre las representaciones mentales de los estudiantes se han dirigido recientemente hacia la elaboración de estrategias eficaces que favorezcan un aprendizaje significativo y estable de los conocimientos científicos (Nussbaum y Novick, 1982; Rowell y Daw. son, 1985; Grimellini et al., 1989; Dreyfus et al., 1990; Duschl y Gitomer, 1991; Brown y Clement, 1992). Sin embargo, los resultados obtenidos han mostrado de forma evidente que los procesos de cambio conceptual son mucho más complejos de lo que se suele pensar (Scott et al., 1992). Uno de los puntos de relativo desfase parece referirse a la falta de una masa crítica de trabajos sobre el proceso de aprendizaje de los estudiantes. Parecen necesarias informaciones sólidas y generalizables sobre la evolución de las dificultades conceptuales y sobre las posibles «etapas de aprendizaje» (Niedderer, 1992). También se conoce poco sobre los estilos cognitivos de los estudiantes, sobre los procesos de toma de conciencia de éstos en su aprendizaje (Hewson y Nennessey, 1992) o de su manera de observar los fenómenos. De hecho, pocos estudios han sido publicados sobre este tema: podemos recordar los trabajos sobre aprendizaje de ideas básicas referentes a los circuitos eléctricos y la electrostática (Schwedes y Schmidt, 1992; Fisher y von Aufschnaiter, 1992; Niedderer e Goldberg, 1993), sobre estructura de la materia (Scott, 1992) y sobre comportamiento de los gases (Sere, 1992) y, por último, los trabajos de Hennessey (1991), sobre el status de las representaciones de los estudiantes. Acompañando paso a paso el cambio de los modos de ver de los estudiantes, estos tipos de trabajos nos dan pistas sobre posibles caminos de menor resistencia y también sobre eventuales asimilaciónes parciales (Grimellini et al., 1989; Dykstra, 1992) que parecen favorecer un aprendizaje significativo de las ciencias.

Las representaciones mentales de los estudiantes en relación con los choques en mecánica han sido objeto de estudio durante más de una década, obteniéndose muchos resultados (Mariani, 1987; Ricci, 1987; Grimellini et al., 1988, 1989; Lemeignan y Weil-Barais, 1988, 1989). En particular fueron delineadas las principales 
características de esas representaciones y las correspondientes dificultades de los estudiantes en el aprendizaje de las leyes de conservación de la cantidad de movimiento y de la energía (Lemeignan y Weil-Barais, 1990, 1994; Grimellini et al., 1993).

La contribucion de este trabajo se refiere a la evolución del aprendizaje de algunos estudiantes de segundo grado en relación con las colisiones, de acuerdo con el análisis que emerge de Ios datos de una investigación realizada en la Universidad de San Pablo (Brasil). Iniciaremos nuestra exposición focalizando algunas características de Ia investigación y delineando los resultados generales sobre el camino cognitivo seguido por los estudiantes. Posteriormente describiremos con detalles las etapas y concluiremos con algunas consideraciones didácticas sobre Ia utilidad de los resultados obtenidos.

\section{LA INVESTIGACIÓN Y LOS RESULTADOS GLOBALES}

La investigación está formada por una serie de entrevistas individuales sobre choques en mecánica. La interacción didáctica que se realizaba a través đe la entrevista, de duracion entre 60 y 90 minutos, estaba estructurada con cl objetivo de garantizar la posibilidad de extraer las ideas de los estudiantes y simultáneamente favorecer su evolución (Orquiza, 1994). La entrevistadora (LOC) interaccionaba de varias formas con el estudiante con la finalidad de aclarar sus ideas, desarrollarlas y, posiblemente, iniciar una confrontación con la visión de la disciplina: mostraba experimentos simples o invitaba al estudiante a realizarlos; pedía previsiones y posibles explicaciones sobre los resultados; discutía las respuestas; daba sugerencias y algunas veces pedía al estudiante que hiciera preguntas a las que respondía más o menos brevemente. Una descripción más detallada de la actividad desarrollada por la entrevistadora y su finalidad, se encuentra en Villani y Orquiza (1995).

La muestra objeto de la investigación estaba formada por seis estudiantes de segundo grado, de edades comprendidas entre quince y veintidós años. Estos fueron escogidos entre los conocidos por Ia entrevistadora de acuerdo con un criterio de comodidad y bajo el compromiso de realizar una serie completa de ocho entrevistas sobre física, con el objeto de garantizar la continuidad del trabajo. Este artículo se refiere principalmente al comportamiento de tres estudiantes (PA, FE y NA), cuyas entrevistas fueron totalmente registradas en vídeo. No obstante, nos referiremos también a tres estudiantes más (CE, THA y DA), cuyas entrevistas fueron solamente registradas en audio o no fueron completamente registradas en vídeo.

Desde el punto de vista del conocimiento académico anterior, CE, DA, THA y NA habían estudiado el tema en cuestión, aunque PA y FE no. En particular FE, había terminado Ios estudios en una escuela técnica donde no tuvo ningún estudio sobre física. Desde el punto de vista de la capacidad intelectual, PA, CE y DA podían ser considerados alumnos medios; NA y THA briltantes, y FE, con grandes dificultades.

Los choques analizados experimentalmente presentaban las siguientes características (en el apéndice A están descritos los aparatos utilizados y los experimentos propuestos):

\section{a) El blanco podía estar en reposo o en movimiento.}

b) El proyectil podía tener movimiento de trasiación (en un péndulo y deslizando sobre un raíl) o de rotación y traslación.

c) El choque podía ser central (con péndulos o bolas sobre un raí, o carritos sobre un carril) o bidimensional (realizados libremente sobre una mesa o, a través del aparato de PSSC).

d) Las masas de los cuerpos interactuantes podían ser iguales, diferentes o muy diferentes (cuando el blanco era el piso, una pared horizontal o un obstáculo fijo en una mesa).

e) Las deformaciones que se producían durante los choques podían ser visibles (con bolitas de goma y plastilina) o invisibles (con bolitas de acero o vidrio).

f) El tiempo de interacción podía ser breve o denorado (en el caso de un carrito con un muelle repulsor).

g) Los choques podían ser realizados entre cuerpos de material elástico o inelástico (plastilina).

h) Durante el choque, el sistema podia estar aislado o no.

Además de todo esto los estudiantes debían resolver también problemas escritos y cuestionarios relacionados con situaciones unidimensionales o bidimensionales. Se pueden encontrar mayores detalles sobre Ios estudiantes entrevistados, los experimentos y los problemas utilizados en Orquiza (1994)

El resultado más significativo de la investigación es la caracterización de la evolución de las representaciones mentales del estudiante como un doble proceso fenomenológico y teórico. En éstos se procuraba repetidamente alcanzar la conquista de algunos objetivos comunes, superando dificultades fuertemente dependientes de la situación analizada.

Grosso modo, la primera vertiente del proceso puede ser llamada «aclaración» de la fenomenología experimental: consistente en la modificación de las representaciones mentales y de los correspondientes modelos espontáneos, danđo mayor importancia progresivamente a variables y hechos científicamente significativos. La segunda vertiente, por el contrario, está constituida por el aprendizaje de los principios de conservación, proceso en el que el estudiante construye sus representaciones teóricas y formales partiendo de la introducción, por parte de la entrevistadora, de varias formulaciones de los 
principios de conservación de la cantidad de movimiento, de la energía cinética y de sus condiciones de validez.

En este trabajo describiremos la etapa del aprendizaje fenomenológico, mostrando que el proceso no era lineal y asumía frecuentemente una forma espiral, pasando por sucesivas resistencias y asimilaciones.

Además mostraremos algunas evidencias conectadas con las representaciones formales, que nos permiten sugerir la hipótesis de un proceso global de aprendizaje en dos fases, en el cual los principios de conservación pueden ser asimilados de modo significativo sólo después de la conquista, con un cierto grado de seguridad y estabilidad, de las representaciones fenomenológicas correspondientes.

La evolución de las representaciones teóricas, que en general sigue un camino semejante al de las representaciones fenomenológicas, está analizada, por razones de espacio e inteligibilidad, en otro trabajo (Orquiza y Villani, 1996). Aquí nos limitaremos a comentar los resultados principales.

\section{LA EVOLUCIÓN DE LAS REPRESENTA- CIONES FENOMENOLOGICAS}

La descripción detallada de la evolución de las representaciones fenomenológicas exige la utilización de tres categorías diferentes: dominio, resistencia y asimilación. El dominio de un estudiante de una determinada representación estaba caracterizado por la relativa estabilidad de ésta y por la tendencia a aplicarla continuamente, sin percibir su limitación. La interacción con los experimentos y con la entrevistadora, que colocaba en discusión la validez del dominio, provocaba en el estudiante una resistencia, manifestada por la tendencia a descartar la interferencia. Sólo la presencia de una nueva asimilación, conseguida generalmente con el descubrimiento o la integración de una nueva información, disminuia sensiblemente la resistencia anterior y tendía a transformarse en un nuevo dominio. La rapidez de la asimilación dependía fuertemente de las características de las situaciones analizadas, que eran más o menos trunsparentes debido a la presencia de distractores asociados a ellas.

\section{Las asimilaciónes esenciales}

La evolución de las representaciones fenomenológicas que emerge de los datos puede ser representada como un proceso que parte de un dominio inicial, caracterizado por algunas ideas espontáneas bastante generales que se traducen en alguna tendencia específica, pasa por diferentes tipos de dificultades y se realiza a través de tres descubrimientos esenciales aplicados a los diferentes tipos de experimentos:

Las asimilaciones son: a) La identificación del cambio en el movimiento del blanco y en el del proyectil, como efecto del choque (aistamiento del choque de las otras variables de contexto);

b) El descubrimiento de una relación determinada entre los cambios de velocidad de los cuerpos interaccionantes (percepción de la existencia de leyes);

c) La caracterización del choque como una interacción debida a una deformación, origen de los efectos en los cuerpos interaccionantes (comprensión de la fuerza de interacción).

Pudimos constatar que los diversos tipos de experimentos condicionaban las dificultades del proceso de aclaración de diversas formas. En particular el alcance de las asimilaciónes A) y B), dependía principalmente de varias combinaciones de las características $a$ ), b) , c), d) y g) de los experimentos analizados. Por el contrario la asimilación C) dependía fundamentalmente de las características $d$ ), e), $f$ ) y $g$ ).

Las asimilaciónes A) y B) estaban jerarquizadas, porque eran lógicamente dependientes. La asimilación C) era parcialmente independiente.

Las entrevistas realizadas tenían como objetivo principal averiguar el alcance de A) y B), que se constituy6 en un proceso homogéneo y articulado. Por el contrario, la asimilación de C) fue seguida sólo parcialmente. En parte fue debido a que el subsiguiente proceso de aprendizaje, el de los principios de conservación, no focalizó el análisis y la descripcióninterpretación del estado intermedio (durante el cual sucede la interacción).

\section{Las representaciones y las resistencias iniciales}

Las ideas espontáneas más importantes registradas en este estudio son semejantes a las encontradas y ampliamente documentadas en trabajos anteriores: equivalencia entre fuerza, movimiento, velocidad y aceleración (Viennot, 1979; Villani et al., 1982; Niedderer, 1992), variación del peso durante un movimiento en la vertical (Mariani, 1987; Villani y Pacca, 1990b), identificación de las magnitudes vectoriales únicamente a través de su módulo (Grimellini et al., 1989), choque como suceso instantáneo que separa los movimientos anteriores de los posteriores (Villani y Pacca, 1990a), modalidad única de producción o de transferencia de movimiento (Mariani, 1987), asociación frecuente entre choque y pérdida de «energía» (fuerza, movimiento, inercia) (Mariani, 1987; Ricci, 1987).

Las resistencias iniciales, manifestadas por los estudiantes en el tratamiento de los choques, pueden ser sintetizadas e interpretadas como negativa a aislar el choque de las variables de contexto y a substituir las variables perceptivas por aquéllas científicamente significativas, dando más importancia a los correspondientes sucesos y condiciones. 


\section{INVESTIGACIÓN Y EXPERIENCIAS DIDÁCTICAS}

En los apéndices B y C estân descritas detalladamente las representaciones y las resistencias iniciales.

\section{El choque como interacción [A]}

La evolución de las dificultades y el alcance de las asimilaciónes han sido marcados por la presencia de caminos preferentes, asociados a las características de Ios experimentos. Además, las asimilaciónes sólo se hicieron definitivas después de haber sido conseguidas a través de un buen número de caminos.

Las asimilaciones del choque (A), como causa de las modificaciones de los movimientos de los cuerpos que interaccionan, siguen caminos diferentes en cuanto a la rapide $z$ del descubrimiento y en cuanto a la presencia de etapas intermedias, dependiendo del tipo de experimento. Por ejemplo, cuando ambos cuerpos estaban dotados de un movimiento inicial, la asimilación era directa, pero la asimilación anterior no era transferida directamente en el caso en el que el blanco estaba en reposo; en esta situación el proceso era articulado en dos etapas sucesivas: la asimilación del movimiento del blanco como efecto del choque y la asimilación del cambio de movimiento del proyectil como efecto de la acción del blanco. En la apéndice $D$ está descrita con mayores detalles la asimilacion del choque en varias situaciones.

\section{La relación entre las velocidades del proyectil y del blanco [B]}

El descubrimiento del choque como suceso singular, al menos en las situaciones más simples y con menor «perturbación», parece ser el punto de partida para elaborar un nuevo conocimiento relativo al experimento. La asimilación de la relación entre proyectil y blanco puede ser sintetizada a través de algunos pasos esenciales. El primer paso era conseguido implícitamente en el uso del modelo de transmisión: de hecho el movimiento perdido del proyectil era transferido totalmente al blan$\mathrm{co}$, salvo en el caso en que hubiese pérdida de energía durante el choque. Fue más difícil aislar la relación entre los cambios de velocidad del proyectil y del blanco en el caso de rodamiento. En los choques contra superficies vertical y horizontal, era simple observar el movimiento del proyectil y el cambio de este movimiento, pero era extremamente difícil reconstruir la fenomenología completa, focalizando el movimiento (invisible) del blanco y su relacion con el del proyectil. Mayores detalles pueden ser encontrados en el apéndice $\mathrm{E}$.

\section{La deformación durante el choque $[\mathrm{C}]$}

La conquista de una visión del choque como una interaccion, caracterizada por la deformación de los materiales que interactuaban, dependía mucho del tipo de experimento analizado. Cuando el choque era entre bolitas de goma o de plastilina, era casi inmediato que los estudiantes percibieran la existencia de una deformación, la diferencia entre una deformacion permanente y temporal, y la relación entre los tipos de deformaciones y el efecto en el movimiento del proyectil y del blanco. Pero esta percepción no constituía la asimilación de una relacion significativa, porque todos los estudiantes se negaban a admitir la existencia de una deformacion (invisible) en las bolitas de acero. Ninguno de los estudiantes consiguió, por sí solo, invertir la relación inicial entre la percepción de una deformación y la modificación del movimiento (de los cuerpos interactuantes) haciéndola necesaria y biunívoca. Cuando la entrevistadora sugirió esta idea, los estudiantes más brillantes asimilaron rápidamente (de forma provisional) aplicándola a las distintas situaciones.

Una idea importante pero poco desarrollada durante la entrevista fue la de la necesidad de una deformación en ambos cuerpos interaccionantes. De hecho, la asimilación definitiva de C) implicaba el descubrimiento de la relación entre deformación y fuerza de interacción, y la asociación de las asimilaciónes A), B) con C) lleváa necesariamente a concluir que ambos cuerpos debían deformarse, incluso cuando estaban compuestos de materiales con diferentes capacidades de deformación. Desgraciadamente tenemos pocos indicios sobre la evolución de las ideas de los estudiantes y sobre las correspondientes dificultades encontradas por ellos.

\section{LAS DIFICULTADES DE LAS REPRESENTACIONES FORMALES}

La idea de la existencia de fases en el desarrollo cognitivo de los estudiantes es muy atractiva, pero generalmente choca con la evidencia experimental de avances y retrocesos, y principalmente de caminos no lineales. También en nuestro caso hubo abundantes situaciones de asimilaciónes parciales seguidas de su abandono más o menos local. Aún nos parece posible recuperar la idea de un aprendizaje en dos fases partiendo de la constatación de las dificultades iniciales en la asimilación de los principios de conservación. En general, la idea de buscar una relación cuantitativa entre las variaciones de las velocidades del proyectil y del blanco, antes y después del choque, y de considerar los sistemas de los cuerpos interactuantes como una unidad significativa, caracterizada por variables globales, adquiría un significado intrínseco para el estudiante sólo después de que el choque era separado del contexto y era reconocido como acontecimiento clave, diferente de la situacion inmediatamente anterior y posterior.

La estrategia didáctica de la entrevistadora, principalmente en las primeras entrevistas, no era la de completar el aprendizaje cualitativo de la fenomenología de los choques antes de introducir los principios de conservación. En varios casos, la idea de una relación cuantitativa, dependiente de la razón entre las masas, entre las variaciones de las velocidades del proyectil y del blanco, fue introducida y aplicada ya en la segunda o en la tercera entrevista. Sin embargo, el comportamiento sub- 
siguiente del estudiante no parecía hacer referencia a esta información, más o menos construida con la entrevistadora, como a un instrumento de comprensión e interpretación de los choques. Varios ejemplos nos parecen muy significativos.

\section{Interpretación de algunos ejemplos}

En el caso de CE, las conservaciones de la energía y de la cantidad de movimiento fueron introducidas y discutidas en varios ejemplos en la tercera entrevista. No obstante, cuando en la entrevista siguiente le fue pedido recapitular cada uno de los experimentos vistos anteriormente, las referencias a los principios de conservación fueron muy vagas. Sólo la ayuda de la entrevistadora consiguió volver a colocar en orden el conocimiento adquirido anteriormente. La dificultad de entrar en la perspectiva de la conservación fue en seguida confrontada varias veces. La entrevistadora, para ayudar a la estudiante, había escrito en la pizarra, en una posición muy visible, las dos leyes de conservación. Casi siempre era dificil el discurso de la estudiante en el comienzo, pero después fluía más rápido y coherente, en la medida en que eran recuperadas las características de los experimentos. Nos parece que el hecho de no haber percibido la dependencia entre la clarificación experimental de los fenómenos y el dominio consciente de los principios de conservación y, por tanto, de no haber insistido suficientemente en la primera fase del proceso de aprendizaje, sea una de las razones para la poca estabilidad del conocimiento alcanzado por la estudiante.

El caso de THA fue en parte semejante, sin embargo, st aprendizaje fue más rápido: de hecho, en su quinta entrevista reencontramos el mismo tipo de interacción, que caracteriz 6 la última entrevista de CE. Las entrevistas siguientes de THA fueron mucho más significativas debido a la naturalidad con que ella se refería a los principios de conservación.

El caso de NA parece muy interesante, porque el estudiante era sin lugar a duda muy hábil para asimitar y estimular las informaciones y las sugerencias de la entrevistadora. Esto le permitió alcanzar y utilizar con éxito los principios de conservación, sobre todo los de la energía cinética, desde las entrevistas iniciales, principalmente cuando podía contar con toda indicación suplementaria de la entrevistadora. No obstante, este nuevo conocimiento no eliminaba el uso del modelo espontáneo de transmisión total o parcial. Cuando, durante la cuarta entrevista, la entrevistadora lo dejó solo ante los problemas de calcular la velocidad del blanco (inicialmente en reposo), que fue alcanzado por un proyectil de masa menor y mayor, NA procuró aplicar la conservación de la cantidad de movimiento identificando la inicial del proyectil con aquella final del blanco (imponiendo entonces, como vínculo implícito, la parada del proyectil). Ante un resultado experimental muy diferente de aquél imaginado, tivo que repasar y reconstruir sus conocimientos teóricos. En la quinta y sexta entrevistas (realizadas con un intervalo de tiempo mayor en relación con las precedentes, debido a las vacaciones), el estu- diante repitio el esquema anterior y tuvo que cambiar de opinión después de la realizacion del experimento correspondiente. Sólo después de la última revisión, en la que se percató del modelo implícito que estaba utilizando, la idea de sistema se le hizo familiar y consiguió usarla coherentemente también en las situaciones siguientes.

En las entrevistas con PA y DA, que fueron las últimas realizadas, la entrevistadora estuvo atenta para garantizar una discusión profunda de cada situación experimen. tal, en parte porque el modo de aprender de los estudian. tes exigía pequeños pasos. Esto no impidió que ellos tuvieran dificultades en el uso de las conservaciones, pero permitió comprender que su introducción era muy natural, siendo aceptada con mayor familiaridad.

Finalmente, el caso de FE representó el ejemplo más clamoroso de vínculo entre la clarificación de la fenome. nología y aprendizaje de las leyes de conservacion. $\mathrm{FE}$ inicialmente poseía una cultura científica muy limitada, y tenía que realizar durante las ocho entrevistas, no sólo el análisis e interpretación de las situaciones propuestas por la entrevistadora, sino también un proceso de apren. dizaje substancial de un nuevo lenguaje caracterizado por una precisión mayor, diferente del espontáneo que ć poseía.

Las transformaciones, en su modo de comprimirse aproximándose al científico y la evolución del proceso de separación del choque del contexto, con la elimina ción de Ias variables irrelevantes, fueron el acontecimiento principal de las ocho entrevistas. Tal evolución paró en la fenomenología bidimensional, porque FE no consiguió conquistar la descomposición del movimiento parabólico en las dos dimensiones y, por tanto, separar la componente horizontal significativa de la vertical. Durante este proceso, las ideas de conservación de Ia energía y de la cantidad de movimiento fueron discutidas con apoyo de la entrevistadora y, en algunos casos, el estudiante consiguio aplicarlas con relativo éxito. Sin embargo, el éxito eventualmente conseguido era muy frágil y en los momentos siguientes de la entrevista era invariablemente negado por afirmaciones completamente diferentes y opuestas. Nos parece legítimo concluir que tales éxitos eran sólo locales y no producían modificaciones substanciales en la manera de ver del estudiante que, sin embargo, consiguió durante las ocho entrevistas modificar substancialmente, en un sentido positivo, su conocimiento experimental. Nuestra opinión se orienta en la interpretación de este comportamiento en términos de fases: el estudiante no había aún alcanzado el mínimo que le permitiese asimilar los principios de conservación a los restantes de su ecologia conceptual (Striker y Posner, 1992).

\section{ALGUNAS CONCLUSIONES DIDÁCTICAS}

Un primer resuitado de esta investigación parece mostrar aspectos bastante paradójicos: por un lado, sugiere la existencia de un camino casi natural de asimilación 
conceptual progresiva y, por otro, indica que este camino depende del análisis de un cierto número de situaciones cruciales, sin las cuales las asimilaciónes permanecen muy frágiles.

El camino que podemos delinear partiendo de nuestra observación e integrando resultados anteriores comienza con el descubrimiento del movimiento del blanco como efecto del choque, separándolo, al menos en parte, de los efectos de variables de contexto, como la gravedad y el rozamiento; rápiđamente es alcanzado el descubrimiento del cambio de movimiento del proyectil y la tendencia de usar sistemáticamente un modelo de transmisión de movimiento. La visión fenomenológica de los choques es completada con la asimilación de la idea de acción del blanco sobre el proyectil, la única que, desde el punto de vista del estudiante, consigue explicar el retroceso del proyectil $y$, con la separación del choque del rodamiento de los cuerpos interaccionantes, hace general la relación entre sus movimientos después del choque y la razón entre sus masas. Finalmente, el descubrimiento de la relación entre la deformación de los cuerpos y el cambio de sus movimientos completa el análisis fenomenológico, dejando totalmente abierto el campo de los principios de conservación, cuyo aprendizajc exigirá nuevamente una serie de asimilaciónes (Orquiza y Villaní, 1996). La fase de aprendizaje de los principios de conservación deberá, de hecho, ser realizada con la asimilación de la idea de sistema, de energía cinética, de cantidad de movimiento, del carácter vectorial de la velocidad y de la cantidad de movimiento y con el respectivo balance escalar y vectorial, que podrá ser expresado de diferentes maneras. Este balance deberá ser extendido también al estado intermedio, con la introducción de los conceptos de energía potencial e interna.

La idea de un camino natural que organiza de modo gradual las dificultades de los estudiantes parece contrastar con la extrema dependencia del aprendizaje de la característica de la situación analizada. La continua presencia de «retrocesos», también referidos a asimilaciones ya aparentemente sedimentadas parece sugerir la necesiđad de promover las asimilaciones en determinadas situaciones en las que el conflicto entre las ideas espontáneas y las nuevas nociones sea particularmente agudo. No pasar por estas situaciones parece hacer particularmente frágil y, probablemente, más fácil abandonar o bien olvidar su asimilación. Concluyendo, pensamos que el descubrimiento de la relación entre el choque y las variaciones del movimiento del blanco debe pasar por una situación que incluya el efecto de la gravedad y đel choque. La variación del movimiento del proyectil debe ser probada en los choques con retroceso y con rodamiento. Finalmente, la relación entre deformación y cambio en el movimiento de los cuerpos interaccionantes debe pasar por el análisis de los choques entre materiales diferentes y particularmente duros. De modo análogo, cada una de las asimilaciones relacionadas con los principios de conservación deberán ser conseguidas superando una correspondiente representación espontánea que ofrece obstáculos o deforma el aprendizaje, principalmente cuando la situación física presenta distractores desfavorables. La idea de continui- dad del movimiento, la tendencia a trabajar solamente con velocidad y con magnitudes escalares, la poca familiaridad con un balance riguroso y principalmente la ausencia de analogías relacionadas con balances vectoriales, son las dificultades expuestas y evidenciadas, que deben ser superadas, principalmente en los choques con blanco en reposo, en los choques bidimensionales y en presencia de blancos «infinitos» (Orquiza y Villani, 1996).

Un segundo resultado importante nos parece que puede ser brevemente resumido en la idea de una correlación estrecha entre el dominio de la fenomenología y aquél de los principios de conservación. Si se quiere que el estudiante consiga sólo una visión superficial de los principios de conservación, probablemente baste con que éste consiga aislar el choque de las condiciones de contorno en cualquier situación, no necesariamente complicada, y aplicar principios de conservación. Pero, si se exige que el estudiante domine los principios de conservación, será necesario gastar un tiempo significativo en el análisis fenomenológico, porque la capacidad de identificar las variables relevantes parece no transferirse de un experimento a otro, debiendo ser reconstruida en cada caso con características diferentes. Nuestro análisis ha focalizado un buen número de estas sjtuaciones. Naturalmente esto no significa que los estudiantes deban completar los procesos de aclaración de todos los tipos de experimentos antes de poder iniciar el aprendizaje de los principios de conservación. La secuencia de las dos fases es principalmente local, en el sentido de que una determinada experiencia o situación puede ser analizada desde el punto de vista de los principios, sólo si su fenomenología ya fue clarificada. Eso no impide que, en algunos casos más complejos, el proceso pueda ser invertido, o sea, la asimilación de una conservación sea provisionalmente transferida de una situación a otra y ayude a clarificar la fenomenología de esta última y a separar el choque del contexto, como sucedió con los choques contra la pared.

Un último resultado alcanzado en este trabajo es la constatación de que el proceso de aprendizaje de los estudiantes estuvo influido significativamente por la intervención didáctica de la entrevistadora. Confrontando los datos de las entrevistas de CE y THA, que fucron realizadas en el inicio del trabajo, con aquéllos de las entrevistas de FE, NA, PA y DA, que fueron realizadas en último lugar, parece que, en el primer caso, la entrevistadora no tenía «estandarizado» sus intervenciones y no había descubierto un camino de menor resistencia, como sucedió, al menos en parte, en la segunda recogida de datos. También el tratamiento diferenciado de los conflictos cognitivos (Villani y Orquiza, 1995), en las dos situaciones, mostró la mayor eficacia de la entrevistadora, al conducir y hacer uso de la situación en favor de una toma de consciencia de las ideas más enraizadas de los estudiantes (Chinn y Brewer, 1993) y de sus tentativas de fuga frente a las dificultades.

Para concluir, debemos resaltar que, por el número limitado de entrevistas sucesivas, nos faltan datos sobre el proceso de coordinación (Grimellini et al., 1993; 
Orquiza y Villani, 1996) de los principios de conservación. A pesár del alcance de esta meta, la cual envuelve el análisis del estado intermedio, que es más complejo que el que comúnmente se espera de la enseñanza de segundo grado (Villani, 1992), nos parece que obtener informaciones sobre las dificultades y los caminos de este proceso será de gran auxilio en la promoción y gestión de la enseñanza de la mecánica.

\section{REFERENCIAS BLBLIOGRÁFICAS}

BROWN, D. y CLEMENT, J. (1992). Classroom teaching experiments in mechanics, en Duit, R., Goldberg, F., Niedderer, H. (eds.). Research in Physics Learning: Theoretical lssues and Empirical Studies, pp. 380-397. Kiel (D): IPN.

(CHINN, A.C. y BREWER, W.F. (1993). The Role of Anomalous Data in Knowledge Acquisition: A Theoretical Framework and Implications for Science Instruction. Review of Educational Research, 63(1), pp. 1-49.

DREYFUS, A., JUNGWIRTH, E. y ELIOVITCH, R. (1990). Applying the "Cognitive Conflict» strategy for conceptual change: Some implications, difficulties and problems. Science Education, 74(5), pp. 555-569.

DUSCHL, R.A. y GITOMER, D.H. (1991). Epistemological Perspective on Conceptual Change: Implications for Educational Practice. Joumal of Research in Science Teaching, 28(9), pp. 839-858.

DYKSTRA, D. (1992). Studying concentual change: Constructing new understandings, en Duit, R., Goldberg, F., Niedderer, H. (eds.). Research in Physics Learning: Theoretical Issues and Empirical Studies, pp. 40-58. Kiel (D): IPN.

FISCHER, H.E. y VON AUFSCHNAITER, S. (1992). The Increase of Complexity as an Order generating Principle of Learning Processes. Case Studies During Physics Instructions, en Duit, R., Goldberg, F., Niedderer, $\mathrm{H}$. (eds.). Research in Physics Learning: Theoreticallssues and Empirical Studies, pp. 225-239. Kiel (D): IPN.

GRIMELLINI, T.N., PECORIB.B., VILLANI, A. y CASADIO, C. (1988). Strategie di Insegnamento e Cambiamento Concettuale: Il Caso degli Urti in Meccanica. Atti VII Convegno Gruppo Nazionale di Didatica della Fisica, pp. 111-142, Pavia (Italia)

GRIMELLINI, T. N., PECORI, B.B., VILLANI, A., CASADIO, C. y PACCA, J.L.A. (1989). Teaching Strategies and Conceptual Change: The Case of Collisions in Mechanics. Paper presented at Annual Meeting of the American Educational Rescarch Assuciation, S. Francisco, CA.

GRIMELLINI, T.N, PECORI, B.B.,PACCA, J.L.A. y VILLANI, A. (1993). Understanding conservation laws in mechanics: Students' conceptual change in learning about collisions. Science Education, 77(2), pp. 169-189.

IIENNESSEX, M.G. (1991). Analysis of conceptual change and status change in sixth graders'concepts of force and motion. Doctoral Dissertation. University of WisconsinMadison (citado en Hewson y Hennessey, 1992.)

\section{AGRADECIMIENTO}

Agradecemos, al Dr. José María de Posada, la fiel y bien cuidada traducción que realizó.

\section{NOTAS}

' Con ayuda parcial de CNPQ (Brasily.

${ }^{2}$ Con ayuda parcial de CAPES/PICD (Brasil).
HEWSON, P.W. y HENNESSEY, M.G. (1992). Making status explicit: A case study of conceptual change, en Duit, $R$, Goldberg, F., Niedderer, H. (eds.). Research in Physics Learning: Theoretical Issues and Empirical Studies, pp. 176-187. Kiel (D): IPN.

LEMEIGNAN, G. y WEIL-BARAIS, A. (1988). Étude de quelques activités de modélisation, en Vergnaud, G., Brousscau, $G$. $y$ Hulin, M. (eds.). Didactique et acquisition de connaissances scientifiques, pp. 229-244. Grenoble: La pensée sauvage.

LEMEIGNAN, G. y WEIL-BARAIS, A. (1989). Enseignement et apprentissage d'un concept par les élèves: la quantité de mouvement en classe de seconde cicle. Bulletin de L'union des Physiciens, 7 I(6), pp. 1013-1030.

LEMEIGNAN, G y WEIL-BARAIS, A. (1994). A deveiopmenta! approach to cognitive change in mechanics. International Journal of Science Education, 16(1), pp. 99-120.

MARIANI, M.C. (1987). Evoluçãodas Concepçōes Espontáncas sobre Colisões. Master Dissertation. Universidad de São Paulo.

NIEDDERER, H. (1992). What Research can Contribute to the Improvement of Classroom Teaching. Proceeding of The International Conference on Physics Teachers' Education, pp. 120-157. Dortmund (Alemania).

NIEDDERER, H. y SCHESTER, H. (1992). Toward an explicit description of cognitive systems for research in physics learning, en Duit, R., Goldberg, F., Niedderer, H. (eds.). Research in Physics Learning: Theoretical Issues and Empirical Studies, pp. 74-98. Kiel (D): IPN.

NUSSBAUM, J. y NOVICK, S. (1982). Aiternative Frameworks, Conceptual Conflicts and Accommodation: Toward a Principled Teaching Strategy. Instructional Science, 11, pp. 183-200.

ORQUIZA, L.C. (1994). Representaçoes mentais e conflitos cognitivos: o caso das colisõos em Mecânica. Tesis doctoral. Faculdade de Educação. Universidad de Sāo Pauto.

ORQUIZA, L.C. y VILLANI, A. (1994). Evolution of mental representations about collisions. Proceedings of the International Conference: Teaching Science and Mathematics for XXI Century, pp. 420-426. Concepción (Chite).

ORQUIZA, L.C. y VILLANI, A. (1996). Aprendizagem dos princípios de conservação em entrevistas didáticas. Investigações em Ensino de Ciências, 1(1), pp. 80-99. 


\section{PSSC (1960). Physics. Boston: D.C. Heath y Company}

RICCI, A. (1987). Uno studio sperimentale sulle rappresentazioni mentali degli studenti in meccanica: il caso deglit urti. Tesi di Laurea. Dipartimento di Fisica. Universidad de Bolonia.

ROWELL, J.A. y DAWSON, C.J. (1985). Equilibration, Conflict and Instruction: A New Class-oriented Perspective. European Journal of Science Education, 7, pp. 331-344.

SCHWEDES, H. y SCHMIDT, D. (1992). Conceptual Change: A case study and theoretical comments, en Duit, R., Goldberg, F., Niedderer, H. (eds.). Research in Physics Learning: Theoretical Issues and Empirical Studies, pp. 188-202. Kiel (D): IPN.

SCOTT, P.H. (1992). Pathways in Learning Science: A case study of the development of One Student's Ideas Relating to the Structure of Matter, en Duit, R., Goldberg, F, Niedderer, H. (eds.). Research in Physics Learning: Theoretical Issues and Empirical Studies, pp. 203-224. Kiel (D): IPN.

SCOTT, P.H., ASOKO, H.M. y DRIVER, R.H. (1992). Teaching for conceptual change. A review of strategies, en Duit, R., Goldberg, F., Niedderer, H. (eds.). Research in Physics Learning: Theoretical lissues and Empirical Studies, pp. 310-329. Kiel (D): IPN.

SÉRÉ, M.G. (1992). Learning by giving and receiving explanations, en Duti, R., Goldberg, F., Niedderer, H. (eds.). Research in Physics Learning: Theoretical Issues and Empirical Studies, pp. 259-277. Kiel (D): IPN.
STRIKE, K.A. y POSNER, G.J. (1992). A Revisionistic Theory of conceptual change, en Duschl y Hamilton (eds.). Philosophy of Science, Cognitive Science and Educational Theory and Practice. Albany, Nueva York: SUNY Press.

VIENNOT, L. (1979). Le raisonnement spontané en dynamique élémentaire. París: Herman.

VILLANI, A. (1992). Conceptual Change in Science and Science Education. Science Education, 76(2), pp. 223-237.

VILLANI, A. y ORQUIZA, L.C. (1993). Representaçōes mentais e Experimentos Qualitativos. Revista Brasilcira de Ensino de Física, 15(1), pp. 74-89

VILLANI, A. y ORQUIZA, L.C. (1995), Conflictos cognitivos, experimentos cualitativos y actividades didácticas. Enseñanza de las Ciencias, 13(3), pp. 279-294.

VIILANI, A. yPACCA, J.L.A. (1990a). Spontancous Reasoning of Graduate Students. International Journal of Science Education, 12(5), pp. 589-600.

VILLANI, A. y PACCA, J.L.A. (1990h). Conceptos espontáneos sobre colisiones. Enseñanza de las Ciencias, 8(3), pp. 238-243.

WEIL-BARAIS, A y LEMEIGNAN, G. (1990). Apprentissage de concepts et modélisation. European Journal of Psychology of Education, 5, pp. 391-437. 


\section{APENDICE A \\ APARATOS EXPERIMENTALES UTILIZADOS EN LAS ENTREVISTAS}

\section{- PENDULO}

\section{Aparato experimental}

El aparato experimental (Fig. 1) estaba constituido por un conjunto de péndulos con bolitas de acero, de $50 \mathrm{~g}$ (denominadas M) y de $100 \mathrm{~g}$ (denominadas $\mathrm{G}$ ) y bolitas de goma $\mathrm{M}$ y $\mathrm{G}$. Una estructura de metal y madera soportaba los péndulos y permitía la substitución de las bolitas. De este modo, era posible realizar varias combinaciones de colisiones entre las bolitas.
Figura 1

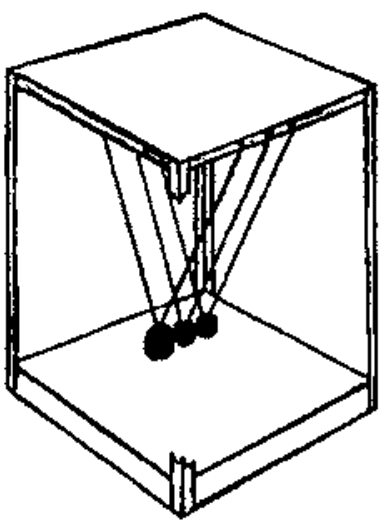

\section{Experimentos}

Tres series de experimentos fueron propuestos a los estudiantes. La serie «p" se refiere a las colisiones entre las bolitas de acero en péndulos. En la serie «b», las colisiones se realizaban con bolitas de goma M y G, en los péndulos. En la serie «m», se proponían dos experimentos imaginarios, donde las bolitas eran de plastilina.

\section{- RIEL}

\section{Aparato experimental}

El aparato experimental (Fig. 2) estaba constituido por un riel metálico plano, con perfil en «U», de $1 \mathrm{~cm}$ de ancho y $1,5 \mathrm{~m}$ de longitud, y de un conjunto de bolitas de acero de $25 \mathrm{~g}(\mathrm{P}), 50$ $\mathrm{g}(\mathrm{M})$ y $100 \mathrm{~g}(\mathrm{G})$.

\section{Experimentos}

l.os experimentos realizados en el riel consistían en colisiones entre bolitas. Varias combinaciones de colisiones podian ser realizadas, alterándose la masa de la bolita incidente y de la bolita blanco. La manera de ser lanzada la bolita incidente también podía ser variada: con un lanzamiento "seco" y rápido, la bolita deslizaba. Sin embargo, cuando era lanzada con un poco de rotación, continuaba rodando hasta alcanzar a ta bolita blanco.

\section{- BOLITAS Y PLACAS DE VARIOS MATERIALES Aparato experimental}

Ei aparato experimental (Fig. 3) estaba constituido por bolitas y placas de plastilina y de goma. Tambien fueron utilizadas "superficies verticales" (pared, por ejemplo) y horizontales (como mesas y bancos) de madera y de cemento.
Figura 2

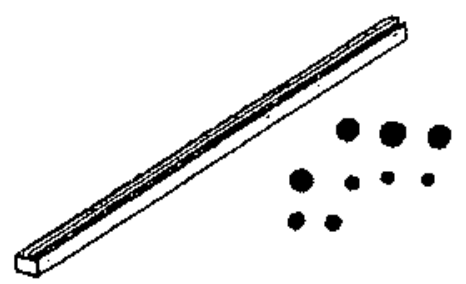

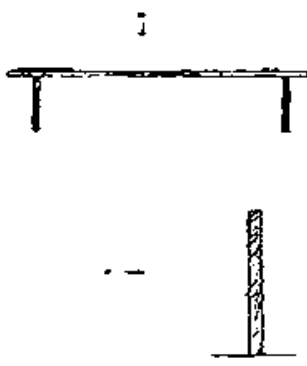

\section{Experimentos}

Las series «m» $y$ «b» de experimentos se refieren a las colisiones elásticas e inelásticas, que fueron realizadas combinándose bolitas de goma y de plastilina con superficies de cemento, de espuma y de madera. 
Cuando el estudiante conseguía aislar los efectos de la gravedad y rozamiento sobre el movimiento del proyectil, el cambio en el movimiento de este último quedaba más claro. El descubrimiento del movimiento del proyectil tendía entonces a transformarse en un dominio en el que los choques eran interpretados con el modelo «transmisión» (total o parcial) del movimiento (o de la fuerza) de un cuerpo al otro (Mariani, 1987). En algunas situaciones, los estudiantes aplicaron este modelo a los choques múltiplos, interpretando el movimiento de cada bolita de acero como efecto del choque de la bolita precedente y catsa del movimiento de ia bolita siguiente. El dominio del modelo de transmisión cra colocado en discusión cuando el blanco era mayor que el proyectil, porque no se comprendía cómo el proyectil podía regresar. Essto no significaba necesariamente la asimilación de la idea de que el blanco actuaba sobre el proyectil, a menos que el estudiante recordase haber estudiado en la escuela el principio de acción y reacción o bien la entrevistadora sugiriese esta idea.

c) En el caso de blanco «infinito» y en el caso de rodamiento inicial del proyectil, el proceso de reconstrucción de la fenomenología era muy complejo y prácticamente se identificaba con la aplicación de una ley física. Cuando el blanco era una pared o una pieza fija en una mesa, debido al choque, la simple observación no era suficiente para reconocer un cambio significativo en las yariables que los caracterizan. Análogamente, cuando el proyecti) era lanzado con un movimiento inicial de rotación resultaba muy difícil distinguir que su movimiento, después de la interacción con el blanco, era debico, en parte, al choque y, en parte, al rodamiento inicial.

\section{APENDICE E \\ LA RELACIÓN ENTRE LAS VELOCIDADES DEL, PROYEC'TX, Y DEL BLANCO}

a) El primer paso era conseguido implícitamente en el uso del modelo de transmisión: de hecho, cl movimiento perdido del proyecti! era transferido totalmente al blanco, salvo en el caso en que hubiese pérdida de energía durante el choque. Pero, como habíamos vișto, este modelo no garantiza la comprensión del comportamiento del proyectil de continuar, parar o regresar, dependiendo de la relación entre su masa y la del blanco. Parece que la variedad de situaciones experimentales y su repetición ayudó o los estudiantes a individualizar y aplicar al menos esta relación. PA, por ejemplo, después de sorprenderse de la observación de retroceso y haber reflexionado sobre ello durante el intervalo entre dos entrevistas, consiguió comprender los choques múltiplos usando la individualidad de la interacción y la relación entre masas para prever los restritados finates, ya sea con bolitas todas igtrales o con bolitas diferentes. De cualquier manera, Ia estabilidad de la conquista no se garantizaba por un exito durante la entrevista; NA por tres veces conquistó y después perdió esta relación, que para estabilizarse necesito de la sustentación teórica de la conservación de la cantidad de movimiento.

b) Más difícil fue aislar la relación entre los cambios de velocidad del proyectil y del blanco en el caso de rodamiento. De hecho, sólo algunos estudiantes consiguieron explicar la diferencia del comportamiento del proyectil cuando éste era lanzado con deslizamiento o con rodamiento. La explicación inicial se refería siempre a la fuerza de lanzamiento o a la velocidad del proyectil, pero los estudiantes entraban en un círculo vicioso cuando confrontaban los choques en el riel con los del péndulo, pues en este último caso el comportamiento del proyectil y del blanco no dependía de la velocidad inicial. Sólo la asimilación de la relación entre la razón entre masas y el movimiento del proyectil permitía a algunos observar que, rápidamente después del choque, el proyectil paraba o retrocedía, incluso durante poco tiempo, del mismo modo que en el caso del deslizamiento. Sin cmbargo, la entrevistadora no insistió mucho sobre este punto y sobre la correspondiente conservación.

c) En los choques contra superficies vertical y horizontal, era simple observar el movimiento del proyectil y el cambio de cste movimiento. Sin embargo, era extremamente difícil reconstruir la fenomenología completa. En reahidad, de un lado era difícil atribuir el cambio de dirección o de sentido del movimiento del proyectil a una interacción con el bianco. ya sea por interferencia de la gravedad o porque el blanco era considerado un obstáculo pasivo mucho más que un agente. Por otro lado, era muy difícil reconstruir el movimiento (invisible) del blanco y relacionarlo con el del proyectil. En este caso, la aclaración de la fenomenología coincidía con el aprendizaje de los principios formales. Sólo la aplicación de la conservación de la cantidad de movimiento podía ayudar al estudiante a aislar el efecto del choque sobre el blanco, relacionando el cambio de su cantidad de movimiento (y, por tarto, de su velocidad) con aquella correspondiente a la del proyectil.

d) Varios estudiantes consiguieron descubrir también algunas regularidades en los choques bidimensionales, notando que la dirección de salida del blanco venía dada por la línea que unía los centros de las bolitas en las cuales, en el caso de las bolitas de acero iguales, el ángulo entre la dirección del proyectil y la del blanco era de aproximadamente noventa grados. 